\title{
Etiologies and Clinical Characteristics of Patients with Macular Hole: A 8-Years Single-Center Retrospective Study
}

Huifang Yue

Shanxi Medical University

Chenyu Liu

Shanxi Medical University

Yunda Zhang

Shanxi Eye Hospital

Lijuan Zhang

Shanxi Eye Hospital

Zhao Gao

Shanxi Eye Hospital

Tao Ma

Shanxi Eye Hospital

Ximei Zhang ( $\nabla$ dr_zhangximei@163.com )

Shanxi Eye Hospital

\section{Research Article}

Keywords: Idiopathic macular hole, Myopic macular hole, Traumatic macular hole, Etiologies, Clinical characteristics, Visual acuity,

Posted Date: October 26th, 2021

DOI: https://doi.org/10.21203/rs.3.rs-969062/v1

License: (a) (1) This work is licensed under a Creative Commons Attribution 4.0 International License. Read Full License 


\section{Etiologies and clinical characteristics of patients with macular hole: a 8-years single-center retrospective study}

Huifang Yue, ${ }^{1 *}$ Chenyu Liu, ${ }^{1 *}$ Yunda Zhang, ${ }^{2}$ Lijuan Zhang, ${ }^{2}$ Zhao Gao, ${ }^{2}$ Tao $\mathrm{Ma},{ }^{2}$ Ximei Zhang ${ }^{2}$

${ }^{*}$ These authors contributed equally to this work

${ }^{1}$ Shanxi Medical University, Taiyuan, China

${ }^{2}$ Shanxi Eye Hospital, Taiyuan, China

Correspondence to Dr Ximei Zhang, Shanxi Eye Hospital, Taiyuan, China; dr_zhangximei@163.com

\section{ABSTRACT}

Background: To investigate the etiologies and clinical characteristics of full-thickness macular hole (FTMH) patients at Shanxi eye hospital of North China.

Methods: Patients diagnosed with FTMH and treated with surgery from 2012 to 2020 were included, and the etiologies and clinical features of different types of MHs were analysed in the 8-years cross sectional retrospective study. Multivariate correlation analysis was used to predict the related factors affecting baseline vision.

Results: A total of 752 cases (776 eyes) were analysed. The top three causes of $\mathrm{MH}$ were idiopathic (IMH, 64.4\%), myopic (MMH, 21.1\%) and traumatic (TMH, 3.7\%). Among these three causes' groups, there were significant differences in sex distribution, age, and baseline BCVA. Female was predominated in IMH and MMH, while it was the opposite in TMH. The age of onset in IMH was older than MMH and TMH. The baseline Logarithm of the Minimum Angle of Resolution (logMAR) best-corrected visual acuity (BCVA) in IMH $(Z=8.9, p<0.001)$ and Others group $(Z=4.0, p<0.001)$ were significantly better than in MMH. In IMH, female patients had younger age, shorter axial length, and poorer baseline BCVA than male, while in MMH there were no significant differences between sexes. Multivariate correlation analysis showed that the smaller hole diameter of IMH, MMH without retinal detachment and younger age in TMH, may resulted in better baseline BCVA.

Conclusions: The most common etiologies in MH were idiopathic, myopic and traumatic, which contributed to the different clinical features. Female was more common in IMH and MMH, and patients with $\mathrm{MMH}$ were 6.5 years earlier than IMH in onset. Therefore earlier monitoring fundus for female and people with high myopia is helpful for early detection and timely treatment.

Key words: Idiopathic macular hole, Myopic macular hole, Traumatic macular hole, Etiologies, Clinical characteristics, Visual acuity,

\section{Background}

Full-thickness macular hole (FTMH) is one of the main causes of central visual impairment, ${ }^{1}$ and the most common etiology is idiopathic macular hole (IMH) associated with vitreous macular traction syndrome. Other causes include high myopia, trauma, diabetic retinopathy (DR), and vitrectomy surgery history, etc. ${ }^{2-4}$ Previous studies have shown that the prevalence of $\mathrm{MH}$ was $0.17 \%$, gradually increasing with age, and bilateral MH was $0.026 \% .^{5}$ In a retrospective study of Tasmanian Australia, the incidence of macular hole was described as 4.05 per 100000 per year, in which the highest was the 70-79 age 
group, IMH, traumatic $\mathrm{MH}(\mathrm{TMH})$, and myopic $\mathrm{MH}(\mathrm{MMH})$ accounted for $87.1 \%, 5.4 \%$ and $2.0 \%$ of the total MH. ${ }^{6}$ Previous studies ${ }^{5}{ }^{8}$ on clinical features of MMH and TMH have been relatively rare compared to IMH, and the results have shown that IMH and MMH had higher incidence in female, while TMH was more common in young male. The studies ${ }^{9-11}$ found the factors affecting baseline vision included hole size in IMH and MMH with retinal detachment (RD), no TMH-related studies were reported.

It is well known that East Asia has the largest number of myopia people in the world and the prevalence of high myopia is up to $6.8-21.6 \%$, while it is $1-4 \%$ in general. ${ }^{12}$ This discrepancy may result in different composition ratios of diverse $\mathrm{MH}$ types in the published articles and there was little literature comparing the clinical features of these types together. ${ }^{4}$ Up to date, we have no found any relevant study in China. Therefore the aim of this study was to explore the etiologies and clinical characteristics of MH and to analyse the related factors affecting baseline vision at Shanxi eye hospital of North China.

\section{Methods}

\section{The inclusion and exclusion criteria of MH patients}

This study was conducted at Shanxi eye hospital, which is the only tertiary eye hospital in Shanxi province of North China, and approved by the ethnic committee of Shanxi eye hospital. The study protocol adhered to the tenets of the Declaration of Helsinki. FTMH patients admitted and performed surgery in our hospital from October 2012 to October 2020 were included. According to the etiologies, they were classified into four groups: IMH, MMH, TMH and Others. The inclusion criteria of MMH were defined as refractive status $>-6.00 \mathrm{DS}$ or axial length $(\mathrm{AL}) \geq 26.0 \mathrm{~mm}$, accompanied with or without RD. If with RD, macular hole was the only one and the extent did not exceed the retinal vascular arch. The cases with ocular trauma history were included in TMH no matter visual loss immediately or lately. Others group included all other recorded causes, such as vitrectomy, the history of DR, retinal vein occlusion, glaucoma, laser photocoagulation and intraocular injection, etc. The cases were excluded when they had peripheral RD caused by peripheral retinal degeneration or refractive media opacity. The priority order of enrollment was Others $>\mathrm{TMH}>\mathrm{MMH}>\mathrm{IMH}$.

\section{Preoperative parameters and examinations}

The collected data included: age of onset, sex, affected eye, the duration of symptom, preoperative intraocular pressure, AL, preoperative best-corrected visual acuity (BCVA), ocular surgery history, general states, diameter of hole-defined as the minimum diameter of the aperture (using Spectralis OCT, Heidelberg, Germany ). VA was expressed using the Logarithm of the Minimum Angle of Resolution $(\log M A R)$, and we referred to the previous studies ${ }^{13}$ and defined counting fingers, hand movement as 2.0, 3.0 respectively.

\section{Statistical analysis}

All data were statistical analysed using SPSS 21.0 (Armonk, NY), in the descriptive analysis of data, continuous variables were expressed as mean \pm standard deviation or median and quartiles, and as numbers and proportions for categorical data. The chi-square test was used to analyse differences among categorical data. For continuous variables, if the variables conform to the normal distribution, we used t-test or ANOVA; otherwise used Mann-Whitney $U$ test or Kruskal-Wallis test to compare differences. Spearman correlation analysis was used to evaluate relationships among factors. $\mathrm{P}<0.05$ was considered significant. 


\section{Results}

In total 776 eyes (752 cases) were enrolled. The eyes were classified into four groups, as shown in Figure 1, IMH, MMH and TMH were the top three causes. All TMH were caused by closed-globe trauma except 3 laser injury cases. In Others group, possible causes included: vitrectomy, scleral buckling, trabeculectomy, branch/central retinal vein occlusion (BRVO/CRVO), proliferative vitreoretinopathy, DR, retinal vasculitis, retinitis pigmentosa, familial exudative vitreoretinopathy, history of vitreous hemorrhage, glaucoma, unknown causes, etc. Among them, the cases with history of vitrectomy accounted for $21.7 \%$, and with DR were $14.5 \%$. IMH and MMH occurred in 51 cases $(10.6 \%)$ and 16 cases $(10.1 \%)$ during the observation.

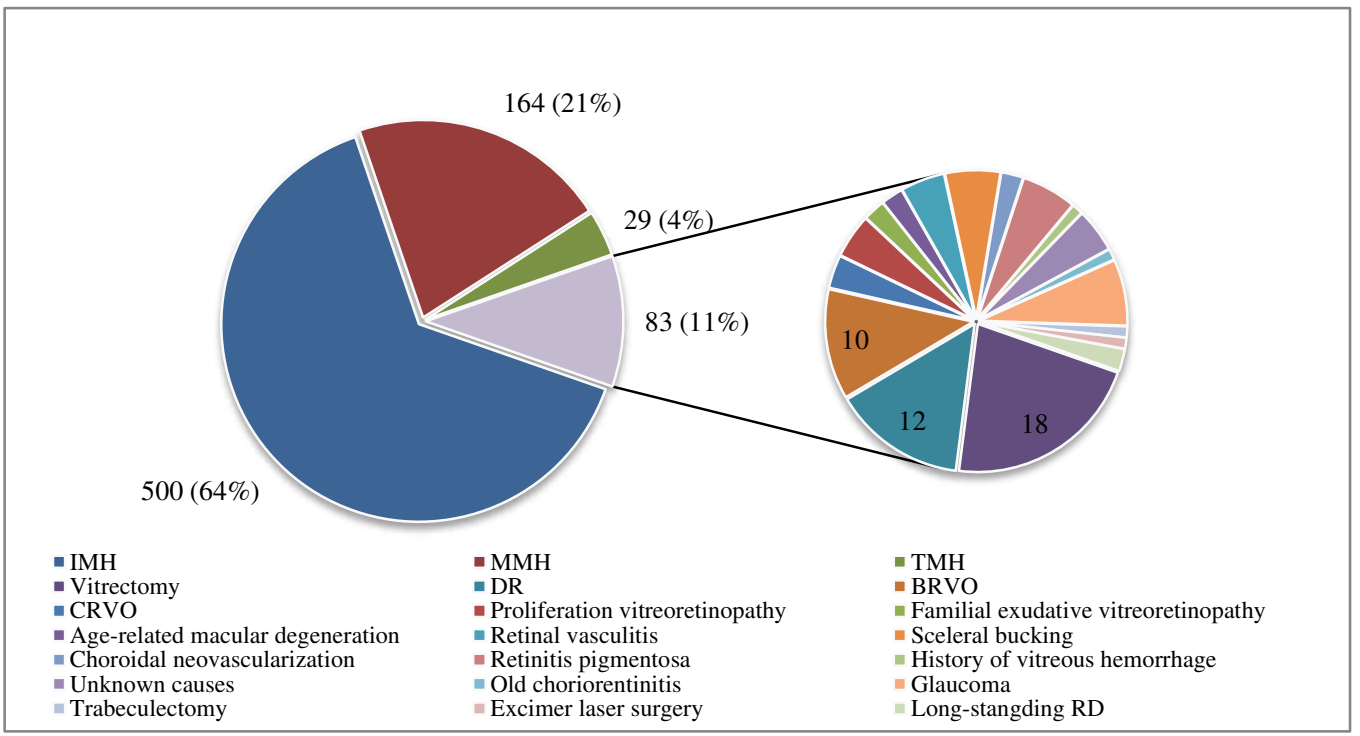

Figure 1 Proportions of eyes and potential causes with diverse types of MHs

The clinical features of IMH, MMH, TMH and Others groups were shown in Table 1. In terms of male-to-female ratio of affecting eyes, male had higher proportion in $\mathrm{TMH}$, while in other groups female were more common. IMH and Others had better VA than MMH. Age of onset was arranged in order of: $\mathrm{IMH}>\mathrm{MMH}>\mathrm{TMH}$, of which $\mathrm{MMH}$ was 6.5 years younger than $\mathrm{IMH}$, and Others group was comparable with MMH in age. Meanwhile, IMH and MMH had different incidence in different age ranges, as shown in Figure 2. Before the sixth decade, the incidence of MMH was higher than that of IMH, but after that, MMH had a lower incidence.

Table 1 Comparisons of clinical characteristics among MHs

\begin{tabular}{lcccccc}
\hline & IMH & MMH & TMH & Others & P & $\chi^{2}$ \\
\hline Patients/Eyes (n) & $482 / 500$ & $158 / 164$ & $29 / 29$ & $83 / 83$ & & \\
Male/Female (n) & $98 / 384$ & $33 / 125$ & $25 / 4_{\mathrm{ab}}$ & $22 / 61_{\mathrm{c}}$ & $<0.001^{*}$ & $66.8^{\dagger}$ \\
OS/OD (n) & $242 / 258$ & $76 / 88$ & $20 / 9$ & $46 / 37$ & 0.091 & $6.4^{\dagger}$ \\
Age (years) & $64.9 \pm 6.8$ & $58.5 \pm 9.0_{\mathrm{a}}$ & $34.3 \pm 13.8_{\mathrm{ab}}$ & $57.4 \pm 14.3_{\mathrm{ac}}$ & $<0.001 *$ & $143.6^{\dagger \dagger}$ \\
Duration(month) & $3.0(2.0,6.0)$ & $3.0(1.0,12.0)$ & $3.0(1.4,10.3)$ & & 0.670 & $0.8^{\dagger \dagger}$ \\
Diameter of hole $(\boldsymbol{\mu m})$ & $518.5 \pm 197.4$ & $572.4 .0 \pm 261.1$ & $609.8 \pm 298.4$ & & 0.053 & $5.9^{\dagger \dagger}$ \\
Baseline BCVA & $1.09 \pm 0.47$ & $1.66 \pm 0.76_{\mathrm{a}}$ & $1.46 \pm 0.88$ & $1.31 \pm 0.36_{\mathrm{b}}$ & $<0.001 *$ & $80.8^{\dagger \dagger}$ \\
LogMAR & & & & & &
\end{tabular}


*,p $<0.05,{ }_{\mathrm{a}} \mathrm{p}<0.05$ compared to IMH, ${ }_{\mathrm{b}} \mathrm{p}<0.05$ compared to MMH, ${ }_{\mathrm{c}} \mathrm{p}<0.05$ compared to $\mathrm{TMH} ; \dagger$

Kruskal-Wallis, ††Pearson's chi squared test

101

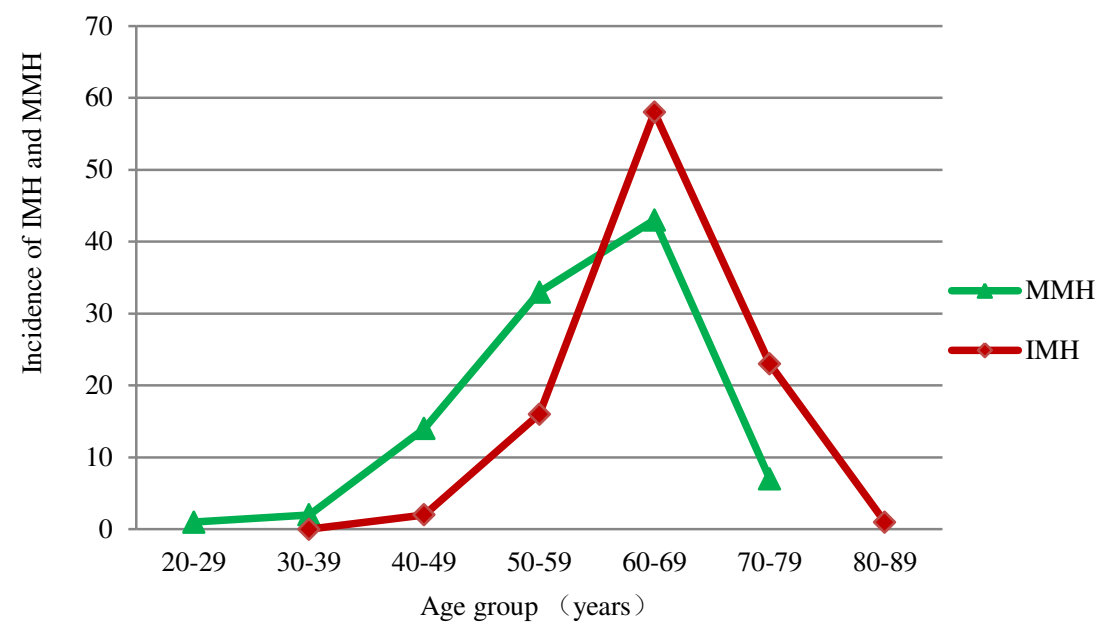

102

Figure 2 The incidence of IMH and MMH in different age groups

To compare the differences of epidemiological characteristics between sexes (Table 2), we found that female had significantly younger age $(Z=3.5, p<0.001)$, worse baseline VA $(Z=2.6, p=0.010)$ and shorter AL $(Z=7.0, p<0.001)$ than male, but there were no differences in diameter of hole and duration in IMH. While there were no differences between sexes in MMH and TMH. For MMH, patients with RD had longer AL $(Z=-2.3, p=0.021)$, shorter duration $(Z=3.2, p=0.01)$ and worse $V A(Z=-6.9, p<0.001)$ than without RD. After comparing the cases of IMH and MMH with or without RD (Figure 3), no difference was found in the baseline BCVA between IMH and MMH without RD.

Table 2 Comparisons of clinical features between sexes among MHs

\begin{tabular}{|c|c|c|c|c|c|c|c|c|c|}
\hline & \multicolumn{3}{|c|}{$\mathrm{IMH}$} & \multicolumn{3}{|c|}{$\mathrm{MMH}$} & \multicolumn{3}{|c|}{$\mathrm{TMH}$} \\
\hline & Males & Females & $\mathrm{P}$ & Males & Females & $\mathrm{P}$ & Males & Females & $\mathrm{P}$ \\
\hline $\operatorname{Eyes}(n)$ & 101 & 399 & & 35 & 129 & & 25 & 4 & \\
\hline Age(years) & $67.2 \pm 6.5$ & $64.5 \pm 6.5$ & $<0.001_{\mathrm{a}} *$ & $59.9 \pm 11.2$ & $58.1 \pm 8.4$ & $0.074_{a}$ & $31.8 \pm 15.4$ & $41.5 \pm 14.5$ & $0.252_{\mathrm{b}}$ \\
\hline fDuration(month) & $3.0(2.0,6.0)$ & $3.0(2.0,6.0)$ & $0.602_{\mathrm{a}}$ & $2.0(0.6,6.0)$ & $3.0(1.0,12.0)$ & $0.311_{\mathrm{a}}$ & $3.0(1.4,3.0)$ & $7.0(0.68,453.0)$ & $0.647_{\mathrm{a}}$ \\
\hline \multicolumn{10}{|l|}{$\mathbf{A L}(\mathbf{m m})$} \\
\hline mean \pm SD & $23.77 \pm 0.90$ & $23.09 \pm 0.96$ & $<0.001_{\mathrm{a}} *$ & $28.99 \pm 2.17$ & $29.14 \pm 2.19$ & $0.745_{\mathrm{a}}$ & $24.14 \pm 1.51$ & $26.78 \pm 2.45$ & $0.027 \mathrm{a}^{*}$ \\
\hline no recorded(n) & 7 & 15 & & 3 & 9 & & 3 & 1 & \\
\hline Baseline BCVA & $0.98 \pm 0.41$ & $1.12 \pm 0.48$ & $0.010 *_{\mathrm{a}}$ & $1.67 \pm 0.90$ & $1.66 \pm 0.72$ & $0.692_{\mathrm{a}}$ & $1.40 \pm 0.81$ & $1.83 \pm 1.41$ & $0.482_{\mathrm{a}}$ \\
\hline Size of hole $(\mu \mathrm{m})$ & $488.6 \pm 188.2$ & $526.1 \pm 200.0$ & $0.088_{b}$ & $568.4 \pm 307.0$ & $573.5 \pm 248.5$ & $0.884_{a}$ & $642.0 \pm 298.1$ & $417.0 \pm 140.7$ & $0.095_{\mathrm{a}}$ \\
\hline With/without RD(n) & & & & $20 / 15$ & $75 / 54$ & $0.916_{\mathrm{c}}$ & & & \\
\hline
\end{tabular}

*, $\mathrm{P}<0.05, \int$ median (quartiles), a, Mann-Whitney U test, b, independent-samples T-test, c, Pearson's chi squared test 

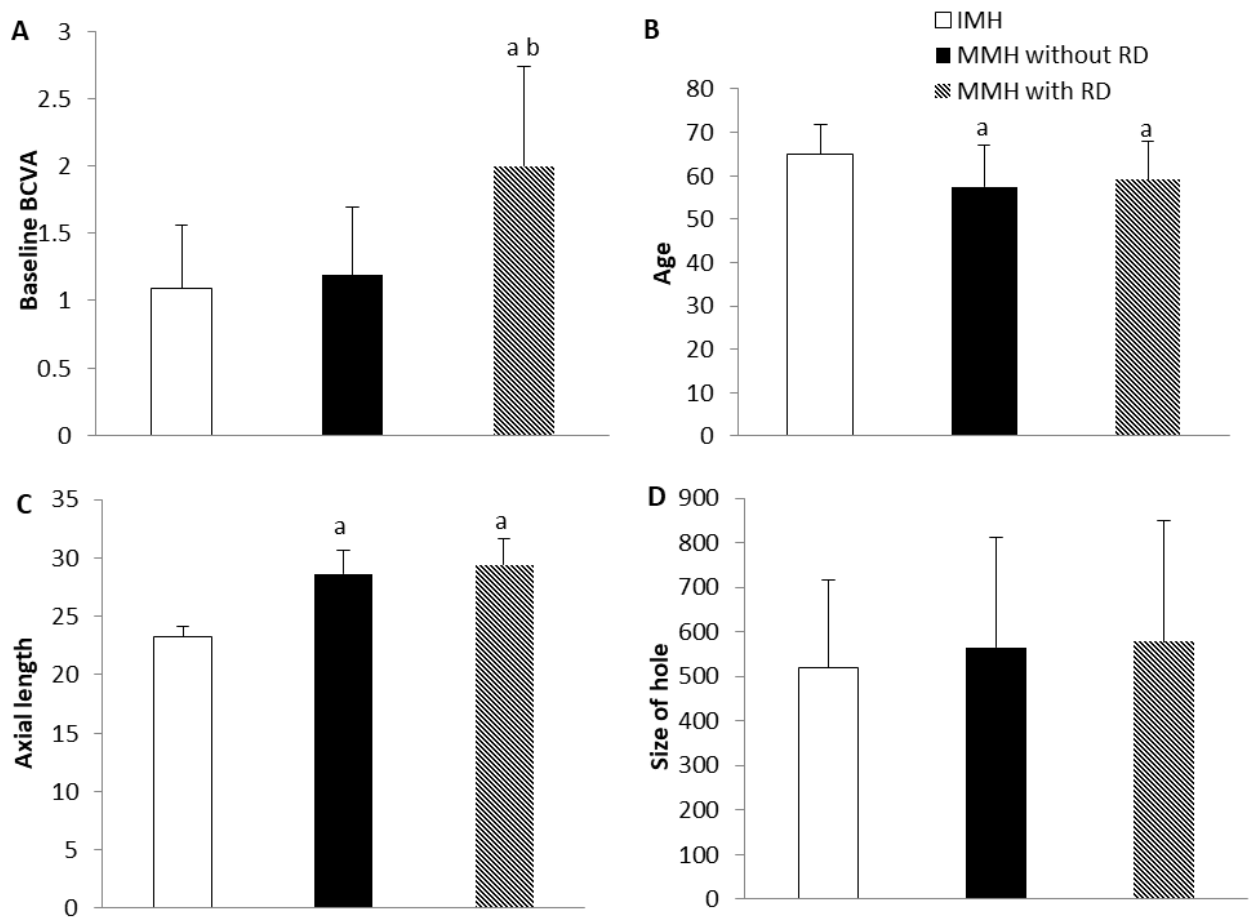

Figure 3 Comparisons of clinical features among IMH, MMH without and with RD. A, B, C, D compare the differences in baseline BCVA, age, AL and hole size of three groups. ${ }^{a} \mathrm{p}<0.05$ compared to IMH, ${ }^{b} \mathrm{p}$ compared to MMH without RD.

Factors related to preoperative VA among MHs are shown in Table 3. The factors affecting VA in IMH were sex, hole size and duration, but the VA only differed between the duration $\leqslant 1$ month and 3-6 month group. After adjusting for possible confounding factors, only hole size was significantly associated with VA $(r=0.386, p<0.001,95 \% \mathrm{CI} 0.313$ to 0.463$)$. Moreover, there was significant correlation between duration and hole size in IMH ( $r=0.303, p<0.001$, 95\%CI 0.223 to 0.387 ). Spearman correlation analysis showed that both AL and with/without RD were correlated with VA in $\mathrm{MMH}$, while after controlling for possible confusing factors, with RD was the only factor correlated with worse VA ( $\mathrm{r}=-0.491, \mathrm{p}<0.001,95 \% \mathrm{CI}-0.590$ to -0.374$)$. The multivariate correlation analysis of $\mathrm{AL}$, hole size, age and VA in TMH showed that there was a significant correlation only between age and VA ( $r=0.446, \mathrm{p}=0.025$, 95\%CI 0.051 to 0.714$)$.

Table 3 Factors affecting preoperative BCVA of IMH and MMH

\begin{tabular}{|c|c|c|c|c|c|c|}
\hline & IMH & $\mathrm{P}$ & statistics & MMH & $\mathrm{P}$ & statistics \\
\hline Male/Female(eye, n) & $101 / 399$ & $0.010^{*}$ & $\mathrm{U}=23459.0 \mathrm{Z}=2.6_{\mathrm{a}}$ & $35 / 129$ & 0.692 & $\mathrm{U}=2354.4 \mathrm{Z}=0.4 \mathrm{a}$ \\
\hline Age (years) & $64.9 \pm 6.8$ & & & $58.5 \pm 9.0$ & & \\
\hline$<49$ & $10(2.0 \%)$ & 0.145 & $\chi^{2}=6.8_{\mathrm{b}}$ & $27(16.5 \%)$ & 0.410 & $\chi^{2}=2.9_{\mathrm{b}}$ \\
\hline $50-59$ & $77(15.4 \%)$ & & & $56(34.1 \%)$ & & \\
\hline $60-69$ & $290(58.0 \%)$ & & & $68(41.5 \%)$ & & \\
\hline $79-79$ & $116(23.2 \%)$ & & & $13(7.9 \%)$ & & \\
\hline$\geq 80$ & $7(1.4 \%)$ & & & - & & \\
\hline \multicolumn{7}{|l|}{ Duration (month) } \\
\hline$\leq 1$ & $111(22.2 \%)$ & $\int 0.004 *$ & $\chi^{2}=17.1_{b}$ & $54(32.9 \%)$ & 0.531 & $\chi^{2}=4.1_{b}$ \\
\hline $1-3$ & $154(30.8 \%)$ & & & $37(22.6 \%)$ & & \\
\hline
\end{tabular}




\begin{tabular}{|c|c|c|c|c|c|c|}
\hline $3-6$ & $132(26.4 \%)$ & & & $25(15.2 \%)$ & & \\
\hline $6-12$ & $70(14.0 \%)$ & & & $28(17.1 \%)$ & & \\
\hline $12-36$ & $27(5.4 \%)$ & & & $12(7.3 \%)$ & & \\
\hline$>36$ & $6(1.2 \%)$ & & & $8(4.9 \%)$ & & \\
\hline Size of hole $(\mu \mathrm{m})$ & $518.5 \pm 197.4$ & $<0.001^{*}$ & $\mathrm{r}=0.419_{\mathrm{c}}$ & $572.4 .0 \pm 261.1$ & $0.047^{*}$ & $\mathrm{r}=0.155_{\mathrm{c}}$ \\
\hline With/Without RD(n) & & & & $95 / 69$ & $<0.001 *$ & $\mathrm{U}=1246.5 \mathrm{Z}=6.9_{\mathrm{a}}$ \\
\hline
\end{tabular}

$*, \mathrm{p}<0.05, \int$ except for the difference between $\leq 1$ and $3-6$ month groups $(\mathrm{p}=0.028)$, no differences were seen between the other groups. a Mann-whitney U test, b Kruskal-wallis test; c spearman's rank correlation analyse.

\section{Discussion}

The present study using 8-years cases analysed the etiologies and epidemiological characteristics of $\mathrm{MH}$, and focused on comparing the discrepancies of age and sex proportion in IMH, MMH and TMH, as well as the different factors affecting baseline VA among them.

In previous epidemiological investigations of Norway and Australia, ${ }^{69}$ the proportion of IMH was larger than this study, accounted for $85.9 \%$ and $87.1 \%$ respectively, and male-to-female ratio was 1:2.2 and $1: 2$, but in other retrospective clinical reports concerning surgical patients, sex ratio was comparable to our data nearly 1:4. ${ }^{14} \quad 15$ Also in the Norway and Australia studies, ${ }^{69} \mathrm{MMH}$ only accounted for $1-2 \%$, while $21.1 \%$ in our study, the reason for this discrepancy probably is that we included MMH with retinal detachment (MHRD), about 57.9\% of all MMH. But even though MHRD were discarded, MMH still accounted for nearly $10 \%$, higher than previous reports. The higher prevalence of myopia in East Asia like China attributed to the result of different MH proportion. ${ }^{12}{ }^{16}$ In previous studies ${ }^{9}$ on clinical surgical patients, TMH represented 3\% of MH, which is consistent with our study, but for those studies $^{17}$ including all TMH cases, TMH accounted for $5-8.2 \%$, the difference for those who less than 24 years old with diameter of hole $<0.2 \mathrm{DD}$ have more chance of achieving spontaneous closure. ${ }^{17}$ Therefore, we only included surgery patients with a significant decrease in VA or a trend of gradual enlargement of the hole during follow-up. $\mathrm{TMH}$ was called the second largest $\mathrm{MH}{ }^{7}$ however in consideration of the result of $\mathrm{MH}$ proportions in our study and spontaneous closure in $\mathrm{TMH}$, the accuracy of the above study need to be further verified.

IMH was older than MMH and TMH, the distinctions of the three types of MH at the age of onset may be related to their underlying pathogenesis. Both IMH and $\mathrm{MMH}$ are complications during the process of posterior vitreous detachment (PVD) which is the consequence of the interaction between vitreous liquefaction and progressive weakening of the vitreoretinal adhesion. ${ }^{18}$ In general, the posterior vitreous cortex initially detaches at the paramacular area and extends to the perifoveal area and then to the optical disc, finally a complete PVD develops, and this inevitable process changes with age. ${ }^{18-20}$ IMH is caused by vitreomacular traction (VMT) which is characterized by aberrant PVD and accompanied by anatomic distortion of the foveal, whereas secondary $\mathrm{MH}$ is caused by other pathological characteristics other than VMT. ${ }^{1}$ The axial elongation and the formation of posterior scleral staphyloma in high myopia accelerate the vitreous liquefaction and its instability, which results in abnormal PVD that has more likely to develop $\mathrm{MH}$, and the greater degree of refraction and the longer AL, the earlier the PVD occurs. ${ }^{21} 22$ Although the axial elongation contributes to the earlier occurrence of $\mathrm{MMH}$, there was no correlation between age and AL in our study. Furthermore, in 
addition to the effect of PVD on formation of FTMH, lower concentration of collagen, protein and hyaluronic acid can prompt the MH development. ${ }^{23}$

The exact mechanism of TMH following blunt trauma is still controversial, it is generally believed that the blunt trauma leads to foveal tissue loss caused by anteroposterior vitreous traction on the fovea. A sudden decrease in the globe's anterior-posterior diameter causes a equatorial expansion of globe, resulting in horizontal and tangential forces and splitting of the retinal layers at the fovea. ${ }^{24}{ }^{25}$ While Rossi et $\mathrm{al}^{25}$ found TMH could also occur in non-vitreous eyes, it revealed that damp shockwaves was also responsible for trauma-related retinal lesions. Accidental high-power laser MH is caused mainly by the rapid photo-thermal damage or photodisruptive mechanism. ${ }^{26}$

With respect to the age of $\mathrm{IMH}$, the results of various epidemiological investigations were inconsistent, roughly between 56.2-70.2 years. ${ }^{6}{ }^{27}$ A respective study $^{4}$ of different types of MHs has described that the mean age of MMH was 42 years, younger than our study. The onset of both IMH and MMH changed with age, which is consistent with the changing of PVD. The area of vitreous macular adhesion gradually decreases after 30 years, the stress acting on the foveal could be increased with decreasing of adhesion area, and the incidence of partial PVD with sustained PVD peaks in the sixth decade. $^{28}$ Therefore, the onset of IMH is about 60 years old. PVD studies on MMH have shown that it occurred earlier, ${ }^{19}{ }^{22}$ but the exact time is not known. In our study, the age of MMH was 6.5 years younger than IMH, which may indicate the PVD of high myopia occurred almost 6.5 years earlier than without myopia. Ali et $\mathrm{al}^{27}$ revealed that age was an independent risk factor of IMH, yet both MMH and IMH showed a gradual increase in the proportions of cases with age in our study, so it may also be an important risk factor for the occurrence of MMH. TMH is more common in young male, since ocular trauma mostly occurred in sports or work-related accidents. ${ }^{724}$

Regarding the onset in different sexes, female had higher incidence and younger age than male in IMH and MMH, although there was no statistically significant difference in MMH age. In female, the decreased estrogen affects the connective tissue, which causes the acceleration of vitreous liquefaction, making it earlier in PVD and more quickly in declining of vitreomacular adhesion area, ultimately leading to more and earlier onset in female. ${ }^{29-31}$ Previous studies $^{915}$ on IMH found male had greater AL than female, and no difference in baseline VA between sexes. The reason for the worse baseline VA in female in our study could be that the average hole diameter was larger than male, though this difference had no statistically significant. Similarly, Steel et $\mathrm{al}^{32}$ also noted female tended to have larger size of hole than male. In contrast with IMH, there were no differences in all preoperative parameters between sexes in MMH. AL was different in TMH between sexes since two of three females with retained axial data were high myopia.

Ghoraba et $\mathrm{al}^{4}$ observed the VA in different types of MHs and found there was no difference in baseline VA, which is inconsistent with the result of our study. It might be the MHRD were excluded in their study. After removing the MHRD cases in our study, it can be seen that the vision difference between MMH and IMH was inexistent, with in accordance to the foregoing studies. Our study found that RD was an important factor affecting MMH vision. In TMH, due to the different causes and pathogenesis of ocular injury, macular hole aside, the lesions such as commotio retinae, choroidal rupture and vitreous hemorrhage might be accompanied. ${ }^{24}{ }^{33}$ Ultimately all lesions lead to an uncertain visual function.

At the multivariate level, in IMH, the smaller hole the better baseline VA retained, the shorter duration the smaller hole, but the duration of symptom had no significant correlation with VA, which was in accordance with previous reports. ${ }^{9} 10$ The better baseline VA was more probable achieved in 
eyes without RD in $\mathrm{MMH}$, besides, the longer AL the more probability to be accompanied by RD. The results of previous studies have shown that the occurrence of RD was related to the AL. ${ }^{12}$ It is difficult to determine the correlation between shorter duration and MHRD, due to the inaccurate complaint of duration and the compensatory effect of vision in contralateral eye. In TMH, since the severity of trauma and the damage of fundus other than $\mathrm{MH}$ were hard to evaluate, it possible dose not to determine its correlation between age and VA.

This study analysed the etiologies and epidemiological characteristics of MH in North China, and to our knowledge, this is the first time by reviewing 8 years' cases, focusing on comparisons of clinical characteristics and factors influencing baseline VA among IMH, MMH and TMH. It was unlike to previous studies, our study had a relatively large sample size and a long time span, and especially in MMH contained MHRD with detachment limited within the vascular arch. The current study still had many limitations: this was a retrospective study in one center; part of data of AL was no recorded, fortunately, the small number of missing cases did not affect the results of study; Our study only included cases having performed surgery and did not contain the observed cases, so it might have deviations in determining the ranking of main etiology. The spontaneous closure rate of TMH might be higher comparing to IMH and $\mathrm{MMH}$, and it could reach the highest-50\% in children as Miller et al ${ }^{33}$ described, even so, the number of TMH case was smaller than MMH and IMH.

\title{
Conclusions
}

Our data demonstrated that the most common causes of MH were IMH, MMH and TMH, MMH accounted for $21.1 \%$, higher than previous studies. Different pathogenesis of the three types of $\mathrm{MH}$ makes it significant differences in age of onset, sex distribution and vision. Not only age, female was also concerned about the risk factor of IMH and MMH. The MMH was nearly 6.5 years earlier than IMH. Therefore, monitoring fundus condition of myopia eyes earlier is necessary for detection and interventional treatment of lesions early.

\author{
Abbreviations \\ FTMH: Full-thickness macular hole; IMH: Idiopathic macular hole; MMH: Myopic macular hole; TMH: \\ Traumatic macular hole; logMAR: Logarithm of the Minimum Angle of Resolution; BCVA: Best-corrected visual \\ acuity; DR: Diabetic retinopathy; RD: Retinal detachment; AL: Axial length; BRVO: Branch retinal vein occlusion; \\ CRVO: Central retinal vein occlusion; MHRD: Myopic macular hole with retinal detachment; PVD: posterior \\ vitreous detachment; VMT: vitreomacular traction \\ Acknowledgements \\ Not applicable.

\section{Authors' Contributions} \\ Study design: XZ, HY; Statistical analysis: HY, CL, TM; Manuscript drafting: HY, CL; Data interpretation and \\ manuscript revising: all authors.

\section{Funding} \\ Technologies R\&D Programs of Health Commission Foundation of Shanxi Province(2011058); Applied Basic \\ Research Programs of Youth Technology Commission Foundation of Shanxi Province(201801D221014)

\section{Availability of data and materials} \\ The analytical data in this study could be obtained from the corresponding author upon reasonable request. \\ Ethics approval and consent to participate
}


The study and data analysis was approved by Shanxi eye hospital Human Research Ethics Committee. The requirement for informed consent was waived due to the retrospective nature of this study.

Patient consent for publication

\section{Competing interests}

None declared

${ }^{2}$ Shanxi Eye Hospital, Taiyuan 030002, Shanxi, China

\section{References}

1 Duker JS, Kaiser PK, Binder S, Smet MD, Gaudric A, Reichel E, et al. The International Vitreomacular Traction Study group classification of vitreomacular adhesion, traction, and macular hole. Ophthalmology. 2013;120:2611-9. 2 Ghoraba H. Types of macular holes encountered during diabetic vitrectomy. Retina. 2002;22:176-82.

3 Kang HG, Han JY, Choi EY, Byeon SH, Kim SS, Koh HJ, et al. Clinical characteristics, risk factors, and surgical outcomes of secondary macular hole after vitrectomy. Sci Rep. 2019;9:19535.

4 Ghoraba HH, Leila M, Zaky AG, Wasfy T, Abdelfattah HM, Elgemai EM, et al. Results of pars plana vitrectomy for different types of macular holes. Clinical Ophthalmology. 2021;15:551-7.

5 Sen P, Bhargava A, Vijaya L, George R. Prevalence of idiopathic macular hole in adult rural and urban south Indian population. Clinical \& Experimental Ophthalmology. 2008;36:257-60.

6 Darian-Smith E, Howie AR, Allen PL, Vote BJ. Tasmanian macular hole study: whole population-based incidence of full thickness macular hole. Clinical \& Experimental Ophthalmology. 2016;44:812-6.

7 Lin C-W, Ho T-C, Yang C-M. The development and evolution of full thickness macular hole in highly myopic eyes. Eye. 2015;29:388-96.

8 Liu W, Grzybowski A. Current management of traumatic macular holes. Journal of Ophthalmology. 2017;2017:1748135.

9 Forsaa VA, Lindtjorn B, Kvaloy JT, Froystein T, Krohn J. Epidemiology and morphology of full-thickness macular holes. Acta ophthalmol. 2018;96:397-404.

10 Kang SW, Ahn K, Ham D-I. Types of macular hole closure and their clinical implications. Br J Ophthalmol. 2003;87:1015-19.

11 Shao Q, Xia H, Heussen FMA, Ouyang Y, Sun X, Fan Y. Postoperative anatomical and functional outcomes of different stages of high myopia macular hole. BMC Ophthalmology. 2015;15:93.

12 Ruiz-Medrano J, Montero JA, Flores-Moreno I, Arias L, Garcia-Layana A, Ruiz-Moreno JM. Myopic maculopathy: current status and proposal for a new classification and grading system (ATN). Progress in Retinal and Eye Research. 2019;69:80-115.

13 Faria MY, Proenca H, Ferreira NG, Sousa DC, Neto E, Marques-Neves C. Inverted internal limiting membrane flap techniques and outer retinal layer structures. Retina. 2020;40:1299-305.

14 McCannel CA, Ensminger JL, Diehl NN, Hodge DN. Population-based Incidence of Macular Holes. Ophthalmology. 2009;116:1366-9.

15 Wang J, Yu Y, Liang X, Wang Z, Qi B, Liu W. Pre- and post-operative differences between genders in idiopathic macular holes. BMC Ophthalmology. 2020;20:365.

16 Chen M, Wu A, Zhang L, Wang W, Chen X, Yu X, et al. The increasing prevalence of myopia and high myopia 
among high school students in Fenghua city, eastern China: a 15-year population-based survey. BMC Ophthalmology. 2018;18:159.

17 Gao M, Liu K, Lin Q, Liu H. Management modalities for traumatic macular hole: a systematic review and single-arm meta-analysis. Current Eye Research. 2017;42:287-96.

18 Kishi S. Vitreous anatomy and the vitreomacular correlation. Jpn J Ophthalmol. 2016;60:239-73.

19 Itakura H, Kishi S. Evolution of vitreomacular detachment in healthy subjects. JAMA Ophthalmol. 2013;131:1348-52.

20 Johnson MW. Posterior vitreous detachment: evolution and complications of its early stages. Am J Ophthalmol. 2010;149:371-82.

21 Tey KY, Wong QY, Dan YS, Tsai ASH, Ting DSW, Ang M, et al. Association of aberrant posterior vitreous detachment and pathologic tractional forces with myopic macular degeneration. Invest Ophthalmol Vis Sci. 2021;62:1-7.

22 Itakura H, Kishi S, Li D, Nitta K, Akiyama H. Vitreous changes in high myopia observed by swept-source optical coherence tomography. Invest Ophthalmol Vis Sci. 2014;55:1447-52.

23 Quinn NB, Steel DH, Chakravarthy U, Peto T, Hamill B, Muldrew A, et al. Assessment of the vitreomacular interface using high-resolution OCT in a population-based cohort study of older adults. Ophthalmology.Retina. 2020;4:801-13.

24 Budoff G, Bhagat N, Zarbin MA. Traumatic macular hole: diagnosis, natural history, and management. Journal of Ophthalmology. 2019;2019:5837832.

25 Rossi T, Boccassini B, Esposito L, Iossa M, Ruggiero A, Ciro T, et al. The pathogenesis of retinal damage in blunt eye trauma: finite element modeling. Invest Ophthalmol Vis Sci. 2011;52:3994-4002.

26 Alsulaiman SM, Alrushood AA, Almasaud J, Alkharashi AS, Alzahrani Y, Abboud EB, et al. Full-thickness macular hole secondary to high-power handheld blue laser: natural history and management outcomes. Am J Ophthalmol. 2015;160:107-13.

27 Ali FS, Stein JD, Blachley TS, Ackley S, Stewart JM. Incidence of and risk factors for developing idiopathic macular hole among a diverse group of patients throughout the United States. JAMA ophthalmol. 2017;135:299-305.

28 Palacio AC, Gupta A, Nesmith BL, Jadav PR, Schaal Y, Schaal S. Vitreomacular adhesion evolution with age in healthy human eyes. Retina. 2017;37:118-23.

29 Steel DHW, Lotery AJ. Idiopathic vitreomacular traction and macular hole: a comprehensive review of pathophysiology, diagnosis, and treatment. Eye. 2013;27:S1-21.

30 Chuo JY, Lee TYY, Hollands H, Morris AH, Reyes RC, Rossiter JD, et al. Risk factors for posterior vitreous detachment: a case-control study. Am J Ophthalmol. 2006;142:931-7.

31 Hayashi K, Sato T, Manabe SI, Hirata A. Sex-related differences in the progression of posterior vitreous detachment with age. Ophthalmology.Retina. 2019;3:237-43.

32 Steel DH, Donachie PHJ, Aylward GW, Laidlaw DA, Williamson TH, Yorston D, et al. Factors affecting anatomical and visual outcome after macular hole surgery: findings from a large prospective UK cohort. Eye. 2021;35:316-25.

33 Miller JB, Yonekawa Y, Eliott D, Kim IK, Kim LA, Loewenstein JI, et al. Long-term follow-up and outcomes in traumatic macular holes. Am J Ophthalmol. 2015;160:1255-58.e1. 